\title{
Uma introdução ao estudo da forma urbana no Brasil
}

\author{
Vítor Oliveira[a] (iD), Bruno Zaitter[b] [iD
}

[a] Universidade do Porto (UPd), Faculdade de Engenharia, Porto, Portugal

[b] Pontifícia Universidade Católica do Paraná (PUCPR), Escola de Belas-Artes, Curitiba, PR, Brasil

Como citar: Oliveira, V., \& Zaitter, B. (2020). Uma introdução ao estudo da forma urbana no Brasil. urbe. Revista Brasileira de Gestão Urbana, v. 12, e20200000. DOI https://doi.org/10.1590/2175-3369.012.e20200000

O número temático da revista Urbe dedicado ao estudo da forma urbana no Brasil reúne um conjunto de textos de alguns dos maiores especialistas Brasileiros em morfologia urbana. Cada um dos textos parte da investigação individual do autor (ou autores) para a pesquisa do seu grupo, da sua universidade e, em alguns casos, da sua cidade e do seu estado. Este texto introdutório pretende fornecer uma estrutura de integração relacionando, de modo necessariamente breve, cada um dos diversos contributos com outras atividades de outros investigadores nacionais e estrangeiros. 0 texto deve ser visto como parte de um processo, parte de uma obra coletiva estendida no tempo, não pretendendo constituir-se como um produto acabado.

\section{O estudo da forma urbana no século XX}

Apresentar de modo abrangente a história disciplinar de uma área do conhecimento num país com a dimensão, a população e a atividade académica do Brasil não é tarefa fácil. Assim sendo, uma tentativa de contribuir para a descrição dessa história disciplinar, como é o caso e o propósito do número temático da Urbe, deve necessariamente partir do trabalho já realizado. Nesse sentido, assumem uma importância chave as sintetizes de Stael Pereira Costa e Cristina Teixeira em dois artigos publicados na revista Urban Morphology (Costa, 2006; Costa e Teixeira, 2014). Oito anos separam os dois artigos, sendo o segundo integrado na coleção The Study of Urban Form in... (Oliveira, 2013), que oferece um conjunto de retratos de diferentes realidades nacionais em diferentes partes do mundo - um desenvolvimento do primeiro artigo publicado na primeira década deste século.

As autoras começam por retratar a investigação morfológica no Brasil da primeira metade e meados do século XX, marcada por um lado pelas obras de historiadores como Sergio Buarque de Holanda e sociólogos como Gilberto Freyre e por outro lado por arquitetos e urbanistas, como Lucio Costa, Sylvio de Vasconcellos e Nestor Goulart Reis Filho. Costa e Teixeira identificam ainda o papel-chave que teve, na morfologia

VO é doutor em Engenharia Civil / Planeamento Urbano, e-mail: vitorm@fe.up.pt

BZ é doutor em Gestão Urbana, e-mail: bruno.zaitter@pucpr.br 
urbana brasileira desta época, o processo de fundação de novas cidades, incluindo capitais de Estado, ora sob influência do modelo de cidade-jardim, ora sob o enquadramento da cidade modernista.

A segunda metade do século XX é claramente marcada pelos anos da ditadura militar (1964-1984), com a disrupção da atividade nas universidades e o declínio da investigação científica. Destas duas décadas de um certo declínio, Costa e Teixeira ressalvam a produção de dois geógrafos - Manuel Correia de Andrade e Milton Santos. A partir de meados dos anos 80, dá-se o regresso ao Brasil de um conjunto de académicos e investigadores que nos anos da ditadura continuaram a sua aprendizagem na América do Norte, Ásia e Europa. Na Europa, o Reino Unido assume um papel de destaque no acolhimento destes investigadores, em particular a Oxford Brookes University (e o professor Ivor Samuels) e a University College London (e o professor Bill Hillier). Do regresso dos académicos brasileiros, importa sublinhar a organização de Seminários sobre Desenho Urbano (SEDUR) em Brasília.

Por fim, Costa e Teixeira identificam ainda quatro linhas de investigação dominantes nas últimas décadas do século XX, com contributos evidentes destes investigadores entretanto regressados: estudos históricos; evolução da população e aspetos funcionais das cidades; assentamentos espontâneos (da qual destacaríamos o trabalho de Carlos Nelson dos Santos); e novas formas urbanas com grande impacto sobre a paisagem.

\section{O estudo da forma urbana na transição do século XX para o século XXI}

\section{Primeira geração}

Nas últimas décadas do século XX começam a emergir e a consolidar-se na morfologia urbana brasileira um conjunto de investigadores que irão assumir uma importância central nos anos seguintes. Destacaríamos quatro destes pesquisadores: Frederico de Holanda (graduado em 1966), Romulo Krafta (g. 1973), Silvio Soares Macedo (g. 1974) e Stael Pereira Costa (g. 1974).

Frederico de Holanda é um dos investigadores que realiza uma parte da sua aprendizagem no exterior. Nas suas duas passagens pela University College London, Holanda é exposto às teorias, conceitos e métodos da sintaxe espacial (ainda emergentes na sua primeira passagem), sendo orientado por Bill Hillier nas suas teses de mestrado e doutoramento (Holanda, 2002). Na sua notável tese de doutoramento, $O$ espaço de exceção, Holanda começa por analisar a sociedade como artefacto, procurando em seguida perceber o espaço de exceção, aplicando finalmente esta estrutura de análise a Brasília. Da sua obra destacaríamos ainda Arquitetura \& urbanidade, que aborda as relações entre espaço e comportamento (Holanda, 2003). Holanda irá ter um papel determinante na disseminação da "lógica social do espaço", a nível nacional e internacional - a organização do $2^{\circ}$ International Space Syntax Symposium (ISSS) será abordada na secção seguinte. Entretanto, na Universidade de Brasília, Holanda funda com Maria Elaine Kohlsdorf e Gunter Kohlsdorf, em meados dos anos 80, o grupo Dimensões Morfológicas no Processo de Urbanização (DIMPU). Este grupo irá atrair ao longo das décadas seguintes um conjunto de investigadores de diferentes gerações, dos quais destacaríamos Valério Medeiros, Rômulo Ribeiro e Gabriela Tenorio.

Para além de descrever detalhadamente o trabalho do DIMPU, o artigo de Holanda publicado no número temático da Urbe explora uma questão-chave em morfologia urbana: o modo como os estudos desenvolvidos por um determinado investigador são influenciados decisivamente pela cidade onde a sua instituição de acolhimento está localizada (Holanda, 2020).

Romulo Krafta desenvolve uma outra linha quantitativa de investigação morfológica. Do mesmo modo que Holanda, também Krafta desenvolveu as suas teses de mestrado e doutoramento no Reino Unido, em Oxford e Cambridge (bem como trabalho de pós-doutoramento em Londres, com Michael Batty). Nesta altura, Krafta publica dois influentes artigos que propõem um conjunto de modelos urbanos e intraurbanos assentes em leituras configuracionais (Krafta, 1994, 1996). Ainda nos anos 90, Krafta funda o Grupo de Pesquisa Sistemas Configuracionais Urbanos na Universidade Federal do Rio Grande do Sul. A influência de Krafta em alguns dos investigadores brasileiros mais ativos da geração seguinte é evidente, destacando-se Mauricio Polidori, Renato Saboya e Vinicius Netto. 
Krafta e Silva (2020) discutem diferentes modos de identificar e medir padrões de forma urbana (considerando abordagens distintas e diferenciando descrições de estado e representações da sua evolução), e aplicam a sua estrutura de análise a um estudo de caso, a cidade de Santa Maria.

Ao contrário de Holanda e Krafta, Silvio Soares Macedo desenvolve toda a sua aprendizagem no Brasil, mais concretamente na Universidade de São Paulo (USP), sendo a sua tese de mestrado orientada por Miranda Magnoli, que terá uma forte influência (assim como Milton Santos) na sua investigação das décadas seguintes. Como no trabalho de Holanda, a investigação de Macedo coloca o enfoque no "objeto" espaços livres (e não nos edifícios). A perspetiva é, contudo, diferente, sendo a abordagem de Macedo mais qualitativa. Desde meados dos anos 80, Macedo edita a revista Paisagem e ambiente, outro importante contributo, mais institucional, para a divulgação das temáticas associadas à forma urbana. Em meados dos anos 90, Silvio Macedo criou o Laboratório da Paisagem e iniciou um conjunto de projetos de investigação sobre o Quadro do Paisagismo no Brasil / QUAPÁ. Neste período destaca-se a publicação de três livros fundamentais: Quadro do paisagismo no Brasil, Praças Brasileiras e Parques urbanos no Brasil, organizando o conhecimento e a história sobre a disciplina do paisagismo e sobre os objetos praças e parques (Macedo, 1999; Macedo e Robba, 2002; Macedo e Sakata, 2002). Uma década mais tarde, Macedo constituiu a Rede Nacional de Pesquisa QUAPÁ-SEL, alterando o anterior enfoque dos espaços livres isolados para os "sistemas" de espaços livres e alargando notavelmente a rede a mais de vinte universidades. Queiroga e Sakata (2020) descrevem em detalhe cada um dos momentos da atividade de investigação da USP e do QUAPÁ-SEL sobre espaços livres, dinamizada por Macedo.

Silvio Macedo influencia não só a geração seguinte de investigadores da USP, como também pesquisadores de outras universidades. Do primeiro grupo fazem parte Eugenio Queiroga, Fábio Mariz e Francine Sakata, entre outros. Queiroga tem uma importância chave, tendo sido vice-coordenador do QUAPÁ-SEL ao longo de 15 anos. Da sua obra destacaríamos a sua tese de doutoramento designada $A$ megalópole e a praça (Queiroga, 2001). O segundo grupo de investigadores inclui Stael Pereira Costa, Eneida Mendonça, Vera Tângari e Jonathas Silva.

Como Holanda e Krafta, também Stael Pereira Costa faz o seu mestrado no Reino Unido. Duas décadas após o mestrado, Costa desenvolve o seu doutoramento com Silvio Macedo. A sua atividade de investigação é claramente influenciada pelas abordagens histórico-geográfica e processual tipológica, sendo a investigadora que mais contribuiu para a disseminação destas abordagens no Brasil. A sua visão sobre estas duas escolas de pensamento foi recentemente sintetizada no influente livro Fundamentos de morfologia urbana (Costa e Gimmler Netto, 2015). Na Universidade Federal de Minas Gerais (UFMG), Costa coordena, juntamente com Cristina Teixeira e Marieta Maciel, o "Laboratório da Paisagem", um centro de investigação com uma forte dinâmica e renovação de gerações, incluindo a presença de jovens investigadores como Manoela Gimmler Netto, Marina Salgado, Simone Safe e Karina Machado.

No artigo publicado no número temático da Urbe, Costa et al. (2020) oferecem uma contextualização do tema no Brasil, avançando em seguida para a forma urbana dos assentamentos em Minas Gerais e da cidade de Belo Horizonte (com o seu singular sistema de ruas em dupla quadrícula) e, por fim, para as atividades da Escola de Arquitetura da UFMG, desde a sua origem há quase um século até as décadas mais recentes.

Antes de avançar para a descrição da atividade de uma segunda geração de morfólogos brasileiros, importa identificar o trabalho de Edja Trigueiro (Universidade Federal do Rio Grande do Norte), de Thereza Carvalho (Universidade Federal Fluminense) e de Denise Antonucci (Universidade Presbiteriana Mackenzie).

\section{Segunda geração}

A atividade de Stael Pereira Costa ao nível da disseminação das teorias, conceitos e métodos das abordagens histórico-geográfica e processual tipológica influenciou não apenas os seus colegas na UFMG, mas também noutras universidades, noutros estados. Destacaríamos a esse nível Eneida Mendonça, na Universidade Federal do Espírito Santo (UFES), e Renato Leão Rego e Karin Meneguetti, na Universidade Estadual de Maringá (UEM).

A atividade de Eneida Mendonça e dos seus colegas na UFES (Mendonça et al., 2014) é descrita por Botechia et al. (2020). As autoras dividem as quatro décadas em análise em três períodos de crescente 
atividade cientifica, identificando influências (Silvio Macedo, para além de Stael Pereira Costa) e pontos de viragem - como a organização da sexta conferência da Rede Lusófona de Morfologia Urbana / Portugueselanguage Network of Urban Morphology (PNUM), em 2017. De facto, mais do que uma conferência, o PNUM de Vitória foi visto por Eneida Mendonça como uma oportunidade estratégica de dinamização do estudo da forma urbana em Vitória e no Espírito Santo, incluindo um conjunto de cursos e workshops nos anos imediatamente antes e depois da conferência, bem como o desenvolvimento de uma série de teses de mestrado e doutoramento. Mais do que um evento singular, Mendonça estruturou um processo bemsucedido.

Renato Leão Rego e Karin Meneguetti desenvolvem investigação morfológica na Universidade Estadual de Maringá (UEM), com forte influência das abordagens histórico-geográfica e processual tipológica. Da sua produção destaca-se As cidades planeadas (Rego, 2009), que reflete sobre a influência britânica na criação de um conjunto de cidades novas no estado do Paraná, e Cidade jardim, cidade sustentável (Meneguetti, 2009), que explora a estrutura ecológica urbana de Maringá - dois temas-chave na investigação destes dois pesquisadores.

Rego et al. (2020) abordam a produção morfológica da UEM, refletindo sobre a adaptação de conceitos de morfologia originalmente formulados em contextos geográficos muito distintos (destacaríamos o contributo de Meneguetti e Costa, 2015, para o desenvolvimento do conceito de cintura periférica), a relação entre investigação morfológica e o projeto urbano e a relação entre forma urbana e ecologia da paisagem. Este artigo é também assinado por uma terceira investigadora, Gislaine Beloto, que tem também contribuído para o desenvolvimento do grupo de Maringá.

Numa linha semelhante, combinando leituras qualitativas com outras mais quantitativas, podemos enquadrar outros grupos de investigação, primeiro em Campinas, depois em Belém. Monteiro et al. (2020) apresentam uma revisão da literatura em morfologia urbana produzida em Campinas ao longo da última década. A revisão coloca em evidência as duas maiores universidades da cidade, a Pontifícia Universidade Católica de Campinas (PUC-Campinas) e a Universidade Estadual de Campinas (Unicamp). Destacaríamos, na primeira, o trabalho de Jonathas Silva e, na segunda, os trabalhos de Evandro Monteiro (em particular, a sua experimentação sobre o ensino da morfologia urbana - Monteiro e Bernardini, 2017) e Silvia Pina. Em ambas, é também evidente a influência de Silvio Macedo e do QUAPÁ-SEL. 0 artigo de Monteiro e seus colegas (Monteiro et al., 2020) conclui dando destaque a uma das linhas de investigação da Unicamp dedicada ao estudo das mutações urbanas.

Cardoso et al. (2020) descrevem a atividade de investigação morfológica desenvolvida na Universidade Federal do Pará (UFPA), e em particular no Grupo de Pesquisa Cidades na Amazónia - onde se destacam Jose Julio Lima, Ana Claudia Cardoso e Roberta Rodrigues. 0 artigo identifica influências (internas e externas), origens e desenvolvimentos e, num olhar mais atento sobre as duas últimas décadas, coloca em evidência algumas singularidades do grupo, como a especificidade dos objetos de estudo - assentamentos informais, muitas vezes com uma forte presença da água enquanto elemento estruturante (e que representam um desafio para a aplicação de teorias, conceitos e métodos da morfologia urbana).

Numa linha mais quantitativa, influenciada por Krafta e por Holanda, descreve-se seguidamente a atividade de três investigadores - Mauricio Polidori, Renato Saboya e Vinicius Netto. Mauricio Polidori tem vindo a desenvolver a sua atividade de modelação urbana no Laboratório de Urbanismo, na Universidade Federal de Pelotas (UFPel). Desde o modelo de simulação de crescimento urbano proposto na sua tese de doutoramento (Polidori, 2005), ao software UrbanMetrics e CityCell, e à plataforma PeopleGrid (apresentado em detalhe por Polidori et al. 2020), a investigação vai no sentido de formular instrumentos dedicados à análise das formas existentes e à avaliação do desenho de formas urbanas futuras.

Renato Saboya tem vindo a desenvolver a sua atividade de investigação na Universidade Federal de Santa Catarina (UFSC), onde também Almir Reis e Ayrton Bueno levam a cabo as suas pesquisas (uma revisão da literatura em forma urbana no estado de Santa Catarina é fornecida por Saboya, 2020). Desde a sua tese de doutoramento, dedicada à conceção de um sistema de suporte à elaboração de planos diretores participativos (Saboya, 1997), Saboya realiza investigação sobre a relação entre vitalidade urbana e tipos edificados e sobre a relação entre tipos arquitetónicos, usos do solo e ocorrência de crimes.

Mantendo um enfoque quantitativo, com presença da sintaxe espacial, destacaríamos o trabalho das últimas duas décadas de Vinicius Netto, desde o seu mestrado orientado por Krafta, passando pelo seu doutoramento, supervisionado por Hillier. Os seus livros Cidade e Sociedade e The social fabric of cities 
(Netto, 2014, 2018) são passos na construção de uma leitura que procura mover-se da forma para a informação, relacionando mentes, sociedade (cognições individuais e sociais) e cidade (forma urbana).

No âmbito da sintaxe espacial importa ainda destacar os trabalhos de Luiz Amorim, na Universidade Federal de Pernambuco (UFPE), e de Lucas Figueiredo, na Universidade Federal da Paraíba (UFPB). 0 primeiro realiza o doutoramento com Hillier, o segundo com Batty. Do seu trabalho conjunto destacaríamos a investigação metodológica sobre "linhas de continuidade" enquanto agregação de várias linhas axiais e como síntese de propriedades topológicas e geométricas. Esta pesquisa foi desenvolvida na primeira década deste século e apresentada em duas conferências da ISSS (Figueiredo e Amorim, 2005, 2007).

Antes de concluir esta sub-secção, e regressando a uma abordagem morfológica mais qualitativa, importa referir o trabalho de Vera Tangari na Universidade Federal do Rio de Janeiro (UFRJ). Com ligação a Silvio Macedo e à rede QUAPÁ-SEL, Tangari tem vindo a desenvolver atividade sobre espaços livres, relacionando morfologia urbana e paisagem, contribuindo para um retrato nacional acerca desta temática (Schlee et al., 2009).

\section{Terceira geração}

Na conclusão deste olhar sobre a investigação morfológica brasileira realizada na transição do século XX para o século XXI, centramo-nos agora na geração de pesquisadores que concluíram a sua graduação no século XXI e/ou que realizaram o seu doutoramento nos últimos anos (ou que estão ainda a realizá-lo).

Começaríamos por destacar o trabalho de Valério Medeiros (graduação em 2001), com um enfoque na sintaxe espacial, sublinhando em particular a sua tese de doutoramento Urbis Brasiliae, orientada por Holanda e Hillier (na sua estadia em Londres). Utilizando a abordagem configuracional e avaliando a acessibilidade topológica dos sistemas de ruas, Medeiros (2013) demonstra, de modo exaustivo e rigoroso, a existência de uma fragmentação estrutural das cidades Brasileiras.

Manoela Gimmler Netto (g. 2006) tem vindo a desenvolver a sua atividade de investigação sob a orientação de Stael Pereira Costa, com um enfoque em abordagens mais qualitativas. Como foi já referido, Fundamentos da morfologia urbana, da autoria de ambas, é uma introdução em língua portuguesa às escolas de pensamento iniciadas por M. R. G. Conzen e Saverio Muratori.

Numa linha de investigação semelhante à de Gimmler Netto integra-se o trabalho de Flavia Botechia. A sua tese de doutoramento, defendida em 2017, tem por tema a permanência dos elementos da forma urbana ao longo do tempo. Em particular, a autora explora a permanência do eixo Maruípe na cidade de Vitória (Botechia, 2017).

Por fim, na sua tese de doutoramento, defendida em 2018, Heraldo Borges analisa a morfogénese do território metropolitano de Vitória, a partir dos padrões e processos de antropização (Borges, 2018).

\section{Momentos-chave}

Como foi referido, desde o final dos anos 70 as teorias, conceitos e métodos da sintaxe espacial fazem parte do debate internacional em morfologia urbana. A viragem do século XX para o século XXI é particularmente ativa para os proponentes da sintaxe espacial. Bill Hillier publica The Space is the Machine e Julienne Hanson lança Decoding Homes and Houses (Hanson, 1998; Hillier, 1996). Em 1997 realiza-se a primeira conferência do grupo, em Londres, e dois anos depois a atenção internacional volta-se para o Brasil - Frederico de Holanda organiza em Brasília o segundo International Space Syntax Symposium (ISSS). Os proceedings da conferência, compostos por cerca de 50 textos, revelam por um lado a capacidade de atração de investigadores de 13 países e, por outro lado, uma presença maioritária do Reino Unido. Para além disso, afirmam também o Brasil, juntamente com os Estados Unidos (e a seguir a Suécia), como o terceiro país mais representado no segundo ISSS.

Se a afirmação institucional do Brasil na comunidade da sintaxe espacial se dá na viragem para o século XXI, o papel do país no International Seminar on Urban Form (ISUF) consolida-se oito anos após a conferência de Brasília. Embora acolhendo investigação no âmbito da sintaxe espacial e de outras abordagens "quantitativas", o ISUF é claramente marcado por duas abordagens, a histórico-geográfica e a 
processual tipológica, com histórias disciplinares que remontam ao final do século XIX e início do século XX (Cataldi et al., 2002; Oliveira, 2019; Whitehand, 1981). Em 2007, Stael Pereira da Costa organiza a 14a conferência do ISUF em Ouro Preto. Ouro Preto 2007 foi uma conferência notável, sob diversas perspectivas: a primeira conferência do ISUF a realizar-se fora da Europa e da América do Norte; a maior conferência do ISUF realizada até então, com mais de 200 apresentações e mais de 300 participantes, e o mais elevado número de países (por comparação com as treze conferências anteriores).

0 terceiro evento chave para a dinamização do estudo da forma urbana no Brasil no século XXI é a criação da Rede Lusófona de Morfologia Urbana (PNUM), enquanto rede regional do ISUF. Embora a proposta de criação do PNUM em 2010 fosse menos ambiciosa, envolvendo apenas investigadores portugueses, a partir da segunda conferência da rede, organizada em Lisboa por Teresa Marat-Mendes, tornou-se evidente que o PNUM deveria ser um projeto que envolvesse os dois países irmãos. Desde então, um conjunto de investigadores brasileiros tem desempenhado um papel-chave no desenvolvimento da rede: Frederico de Holanda, Eneida Mendonça e Renato Leão Rego, coordenando a organização das conferências de Brasília 2015, Vitória 2017 e Maringá 2019; Júlio Vargas, Renato Saboya e Vinicius Netto, enquanto editores da "Revista de Morfologia Urbana", desde o início de 2019; e Renato Leão Rego, Karin Meneguetti e Gislaine Beloto, coordenando o 4o Workshop do PNUM. Para além destes, sublinha-se ainda o contributo decisivo de Stael Pereira da Costa, atual Vice-presidente do ISUF.

\section{Desafios fundamentais para a próxima década}

Apesar do papel de destaque de alguns investigadores brasileiros em instituições internacionais como o ISUF e o ISSS, um dos principais desafios que se coloca à morfologia urbana brasileira é o da internacionalização. De um modo geral, os investigadores brasileiros a trabalharem nesta área do conhecimento publicam pouco em língua inglesa, o que dificulta a sua disseminação por públicos fora do espaço lusófono. A título de exemplo refere-se uma análise dos artigos publicados em três das revistas mais importantes no estudo da forma física das cidades - Urban Morphology, Environment and Planning B e Urban Design International - ao longo dos últimos cinco anos. A análise revela que os investigadores brasileiros publicaram apenas três artigos nestas revistas, neste período.

Outro desafio é o de um debate mais equilibrado entre arquitetos e urbanistas, por um lado, e geógrafos (e eventualmente historiadores), por outro. Costa e Teixeira (2014) referem este desequilíbrio na segunda metade do século XX, e apontam a influência da escola Francesa de geografia quantitativa - centrada em temas sociais, políticos e económicos - como uma das razões para esta reduzida participação dos geógrafos brasileiros no debate sobre a forma física das cidades. Uma década de conferências do PNUM confirma este desequilíbrio disciplinar. Este é também um desequilíbrio que urge contrariar na próxima década.

Um terceiro desafio é o de um maior equilíbrio territorial. Apesar da excelência quer de centros de investigação, quer de investigadores nas regiões Norte, Nordeste e Centro-Oeste, as regiões do Sudeste e do Sul do Brasil apresentam uma maior dinâmica na área do conhecimento da morfologia urbana. Aparentemente, esta constatação está em linha com o que se passa noutras áreas do conhecimento. No entanto, devem ser realizados esforços de dinamização do estudo da morfologia urbana (a nível individiual e institucional) nas três regiões referidas.

Por fim, importa sublinhar a questão da relevância. É necessário um maior esforço no sentido de sublinhar a relevância de cada investigação: qual é o estado da arte "neste" tema e qual é o contributo "desta" investigação para o avanço do conhecimento em morfologia urbana? Estas são questões-chave que merecem respostas tão claras quanto possível. Como refere Whitehand (2012), citando uma frase muito utilizada por Albrecht Penck: when you see the particular, always look for the general.

\section{Referências}

Botechia, F. R. (2017). A forma indelével: estudos morfológicos sobre a persistência elementar em Maruípe, Vitória (Tese de doutoramento). Universidade Presbiteriana Mackenzie, São Paulo. 
Botechia, F. R., Mendonça, E. M. S., \& Pegoretti, M. S. (2020). 0 estudo da forma urbana no Espírito Santo. urbe. Revista Brasileira de Gestão Urbana, 12, e20190273. https://doi.org/10.1590/2175-3369.012.e20190273.

Borges, H. F. (2018). A forma do informe: uma interpretação da morfogênese do território metropolitano da Grande Vitória a partir dos padrões e processos de antropização (Tese de doutoramento). Universidade Presbiteriana Mackenzie, São Paulo.

Cardoso, A. C. D., Lima, J. J. F., Ponte, J. P. X., Ventura Neto, R. S., \& Rodrigues, R. M. (2020). Morfologia urbana das cidades amazônicas: a experiência do Grupo de Pesquisa Cidades na Amazônia da Universidade Federal do Pará. urbe. Revista Brasileira de Gestão Urbana, 12, e20190275. https://doi.org/10.1590/2175-3369.012.e20190275.

Cataldi, G., Maffei, G. L., \& Vaccaro, P. (2002). Saverio Muratori and the Italian school of planning typology. Urban Morphology, 6, 3-14.

Costa, S. A. P. (2006). Brazilian urban morphology. Urban Morphology, 10, 142-144.

Costa, S. A. P., \& Teixeira, M. C. V. (2014). The study of urban form in Brazil. Urban Morphology, 18, 119-127.

Costa, S. A. P., \& Gimmler Netto, M. M. (2015). Fundamentos da morfologia urbana. Belo Horizonte: C/Arte.

Costa, S. A. P., Teixeira, M. C. V., Salgado, M., \& Netto, M. M. G. (2020). A investigação da forma urbana em Minas Gerais, Brasil. urbe. Revista Brasileira de Gestão Urbana, 12, e20200095. https://doi.org/10.1590/2175-3369.012.e20200095.

Figueiredo, L., \& Amorim, L. (2005). Continuity lines in the axial system. In Proceedings of the 5th Space Syntax International Symposium (p. 161-174). Delft: Delft University of Technology.

Figueiredo, L., \& Amorim, L. (2007). Decoding the urban grid: or why cities are neither trees nor perfect grids. In Proceedings of the 6th International Space Syntax Symposium (p. 01-16). Istanbul: ITU.

Hanson, J. (1998). Decoding homes and houses. Cambridge: Cambridge University Press.

Hillier, B. (1996). Space is the machine. Cambridge: Cambridge University Press.

Holanda, F. R. B. (2002). O espaço de excepção. Brasília: Editora UnB.

Holanda, F. R. B. (ed.) (2003). Arquitetura \& urbanidade. Brasília: FRBH Edições.

Holanda, F. R. B. (2020). Inclusão e exclusão em Brasília. urbe. Revista Brasileira de Gestão Urbana, 12, e20190306. https://doi.org/10.1590/2175-3369.012.e20190306.

Krafta, R. (1994). Modelling intraurban configurational development. Environment and Planning B: Planning and Design, 21, 67-82.

Krafta, R. (1996). Urban convergence: morphology and attraction. Environment and Planning B: Planning and Design, 23, 37-48.

Krafta, R., \& Silva, E. L. B. (2020). Detecção de padrões da forma urbana: quadro geral e um caso. urbe. Revista Brasileira de Gestão Urbana, 12, e20190209. https://doi.org/10.1590/2175-3369.012.e20190209.

Macedo, S. S. (1999). Quadro do Paisagismo no Brasil. São Paulo: Edusp.

Macedo, S. S., \& Robba, F. (2002). Praças brasileiras. São Paulo: Edusp.

Macedo, S. S., \& Sakata, F. G. (2002). Parques urbanos no Brasil. São Paulo: Edusp.

Medeiros, V. (2013). Urbis Brasiliae - o labirinto das cidades Brasileiras. Brasília: Editora UnB.

Mendonça, E. M. S., Freitas, J. F. B., Campos, M. M., Prado, M. M., \& Almeida, R. H. (2014). Cidade prospectiva - o projeto de Saturnino de Brito para Vitória. Vitória: EDUFES.

Meneguetti, K. S. (2009). Cidade jardim, cidade sustentável - a estrutura ecológica urbana e a cidade de Maringá. Maringá: EdUEM.

Meneguetti, K. S., \& Costa, S. A. P. (2015). The fringe-belt concept and planned new towns: a Brazilian case study. Urban Morphology, 19, 25-33.

Monteiro, E. Z., \& Bernardini, S. P. (2017). Gincana de urbanismo: um instrumento pedagógico de leitura da paisagem. 6ª Conferência da Rede Lusófona de Morfologia Urbana. Vitória: UFES. 
Monteiro, E. Z., Pina, S. A. M. G., Freire, R. A., \& Turczyn, D. T. (2020). 0 estudo da morfologia urbana na Região Metropolitana de Campinas. urbe. Revista Brasileira de Gestão Urbana, 12, e20190260. https://doi.org/10.1590/21753369.012.e20190260.

Netto, V. M. (2014). Cidade \& sociedade - as tramas da prática e seus espaços. Porto Alegre: Sulina.

Netto, V. M. (2018). The social fabric of cities. Londres: Routledge.

Oliveira, V. (2013). The study of urban form: reflections on national reviews. Urban Morphology, 17, 85-92.

Oliveira, V. (ed.) (2019). JWR Whitehand and the historico-geographical approach to urban morphology, Cham: Springer.

Polidori, M. C. (2005) Crescimento urbano e ambiente: um estudo exploratório sobre as transformações e o futuro da cidade (Tese de doutoramento). Universidade Federal do Rio Grande do Sul, Porto Alegre.

Polidori, M. C., Peres, O. M., \& Tomiello, F. (2020). Modelagem e morfologia urbana com o PeopleGrid. urbe. Revista Brasileira de Gestão Urbana, 12, e20190234. https://doi.org/10.1590/2175-3369.012.e20190234.

Queiroga, E. F. (2001). A megalópole e a praça: o espaço entre a razão de dominação e a ação comunicativa (Tese de doutoramento). Universidade de São Paulo, São Paulo.

Queiroga, E. F., \& Sakata, F. G. (2020). A rede de pesquisadores reunidos por Silvio Macedo sob o Lab QUAPÁ e os estudos de sistemas de espaços livres e formas urbanas no Brasil. urbe. Revista Brasileira de Gestão Urbana, 12, e20190264. https://doi.org/10.1590/2175-3369.012.e20190264.

Rego, R. L. (2009). As cidades plantadas - os Britânicos e a construção da paisagem do norte do Paraná. Londrina: Humanidades.

Rego, R. L., Meneguetti, K. S., \& Beloto, G. E. (2020). Morfologia urbana como instrumento projetual. urbe. Revista Brasileira de Gestão Urbana, 12, e20190266. https://doi.org/10.1590/2175-3369.012.e20190266.

Saboya, R. T. (1997). Concepção de um sistema de suporte à elaboração de planos diretores participativos (Tese de doutoramento). Universidade Federal de Santa Catarina, Florianópolis.

Saboya, R. T. (2020). 0 estudo da forma urbana em Santa Catarina, Brasil. urbe. Revista Brasileira de Gestão Urbana, 12, e20190269. https://doi.org/10.1590/2175-3369.012.e20190269.

Schlee, M., Nunes, M. J., Rego, A., Rheingantz, P., Dias, M. Ângela, \& Tangari, V. (2009). Sistema de espaços livres nas cidades Brasileiras - um debate conceitual. Paisagem e Ambiente, 26, 225-247.

Whitehand, J. W. R. (1981). Background to the urban morphogenetic tradition. In J. W. R. Whitehand (ed.) The urban landscape: historical development and management; papers by MRG Conzen. Londres: Institute of British Geographers / Academic Press.

Whitehand, J. W. R. (2012). Issues in urban morphology. Urban Morphology, 16, 55-65.

Editores convidados: Vitor Oliveira (Universidade do Porto, Portugall) e Bruno Zaitter (PUCPR, Brasil) 


\section{Errata}

No artigo "Uma introdução ao estudo da forma urbana no Brasil" com número de DOI: 10.1590/2175-3369.012.e20200000, publicado no periódico Urbe - Revista Brasileira de Gestão Urbana, 2020, 12, e20200000, no cabeçalho, na página 1,

Onde se lia:

Editorial: 0 Estudo da Forma Urbana no Brasil (Editores convidados: Vítor Oliveira e Bruno Zaitter)

Leia-se:

Seção Temática: O Estudo da Forma Urbana no Brasil (Editores convidados: Vitor Oliveira e Bruno Zaitter) 